\title{
Spatial interaction and security: A review and case study of the Syrian refugee crisis ${ }^{1}$
}

Michael J Frith, Miranda Simon, Toby Davies, Alex Braithwaite and Shane D Johnson

\section{ABSTRACT}

Sir Alan Wilson's ideas and approaches have been highly influential in a number of fields within urban and regional modelling, including migration, transport and economics. Latterly, a substantial volume of research has explored the application of similar ideas to new problems, and at larger scales, many of which relate to major global challenges with significant policy implications. In this paper, we first review some of Alan's contributions in this area, focussing on his work on crime and security and its relationship with other dynamic phenomena. Following this, we present the results of some original empirical work concerning forced migration associated with the current Syrian refugee crisis. This work is directly inspired by Alan's work, and shares his ambition of contributing to a pressing policy challenge. In our model, which applies a spatial interaction framework, we examine the flows of migrants forced to leave Syria and the characteristics which influence their choice of destination country. In line with the intuitions of the broader literature, we find that shorter distances, economic prosperity, and cultural similarity (e.g., shared language and historical ties) attract forced migrants. Furthermore, we find that migrants are more likely to favour countries in which the probability of being granted asylum is higher; a finding with potential implications for policy. Contrary to expectation, we find little influence for levels of security in potential host nations (e.g. absence of crime and terrorism). This paper represents a preliminary modelling effort in this area which will be extended in future work incorporating dynamic models.

\footnotetext{
${ }^{1}$ The authors would like to acknowledge support the Army Research Office under Grant Number W911NF-171-0030. The views and conclusions contained in this document are those of the authors and should not be interpreted as representing the official policies, either expressed or implied, of the Army Research Office or the U.S. Government.
} 


\section{INTRODUCTION}

In this paper, we discuss some of Sir Alan Wilson's contributions to the study of crime and security, which is the field in which we have most closely collaborated. We then present the findings of an original empirical study concerned with the Syrian refugee crisis, which is inspired by - and builds directly upon - his earlier work and illustrates the diversity of subject areas to which his approaches continue to be applied. We start by reviewing some of the work in which Alan has been directly involved, discuss how this inspired our work on Syrian refugees, and then present our findings.

Between 2010 and 2015, three of the current authors (Braithwaite, Davies and Johnson) had the pleasure of working on a large interdisciplinary EPSRC-funded project for which Alan was the Principle Investigator. This project - Explaining, Modelling, and Forecasting Global Dynamics (ENFOLDing) - sought to develop and apply mathematical tools to understand the global dynamics and inter-dependencies of four systems: trade, migration, security (including crime), and overseas development aid. One of the central themes of this project was the desire to apply the modelling approaches pioneered by Alan - which had been successful in urban and regional modelling - at both a larger scale and in novel domains. Crime and security were one such domain and, in what follows, we discuss some of the research in this area conducted as part of that project, as well as some of the work concerned with migration which directly influenced the original research that we present in this paper. We direct the reader interested in more details of the wider research conducted within ENFOLDing to two edited collections that describe the project. The first (Wilson, 2016a) adopts a more mathematical orientation, while the second (Wilson, 2016b) is primarily concerned with policy implications.

\section{Crime and Security}

Just like many other phenomena, incidents of crime - and threats to security more generally - exhibit clear spatial patterns (e.g., Johnson, 2010; Weisburd, 2015; LaFree et al., 2012). In the ENFOLDing project, we explored the spatial patterns of a number of crime and security phenomena, at a range 
of geographic scales, with a focus on those which invited analogy with the other systems under consideration. Some analyses used novel data, while others used novel mathematics, and indeed many used both.

The first example we will discuss concerned the sudden onset riots, which occurred in London over the five-day period between August $6^{\text {th }}$ and $10^{\text {th }} 2011$. Unprecedented in scale for the UK in recent times, these were estimated to have caused more than $f 250$ million damage (Met Police, 2012) and led to over 3,000 arrests. Unlike pre-planned protests, the riots could have happened anywhere, which presented challenges to their policing. With a few exceptions (e.g., Abudu-Stark et al., 1974), previous empirical research on riots had tended to examine patterns at the city scale (e.g., Midlarsky, 1980; Myers, 2010), which provides only limited understanding of the precise spatial patterns, how they evolve, and how law enforcement might police them. To provide more insight, we conducted a series of studies at much smaller spatial scales to examine whether the spatial pattern of the riots was random, as would be expected by some perspectives (Le Bon, 1960), or whether there were distinct spatial patterns. Our findings provided clear support for the latter, with there being statistical evidence of spatial clustering at a range of spatial scales including census areas and 400 metre grid squares (Baudains et al., 2013c; Baudains et al., 2013a). Evidence of spatial contagion was also found, whereby rioting appeared to spread to nearby locations (Baudains et al., 2013a; Baudains et al., 2013b).

Having established that patterns were not random, the next stage of the research sought to examine the spatial behaviour of offenders; that is, why they chose to riot in the locations that they did. We tested hypotheses identifying the utility of certain locations as a function of their properties, which is a familiar problem in urban and regional modelling. Two approaches were taken to do this, one using a statistical random utility framework (McFadden, 1974) to examine the target choices of offenders, relative to their home location, and a second employing a more mathematical framework. These studies (Baudains et al., 2013a; Baudains \& Johnson, 2018) showed that, all else equal, offenders 
tended to engage in the riots in areas that were closer to their home location, in areas with higher levels of deprivation, in areas with more retail facilities, and in areas that had experienced rioting in the previous 24 hours.

These findings supported suggestions made elsewhere that the London riots had been largely acquisitive in nature, with looting a dominant driver. This invited a natural analogy with retail modelling: if rioters sought similar rewards to shoppers, then models which had been successful in that context might be adapted in order to understand the large-scale behaviours of rioters. Alan's previous work in the retail context represented a canonical example of spatial interaction modelling (Wilson, 1970), one goal of which is to estimate flows of some quantity (e.g., money or people) between a system of locations. In particular, given stocks at origins, and the utility characteristics of destinations, an entropy-maximising approach can be used to calculate the most likely inter-location flows. Subsequent work (Wilson, 2008) showed how this approach could be combined with other models in order to represent evolving flows in dynamical systems.

The riot scenario could be framed in these terms: the populations of residential areas constituted the 'stocks' of potential rioters, and the utility of potential targets could be modelled in terms of their retail volume and travel distance. This was combined with a model of riot involvement based on the 'SIR' epidemiological paradigm (Anderson and May, 1992) to produce a dynamic model of riot activity across the city, with a simple model of police activity also included (Davies et al, 2013). The model was capable of reproducing many of the empirical features of the London activity, such as distance decay and agglomeration at certain locations, and therefore constitutes a dynamic, macrolevel equivalent of the previous target-choice research. In particular, the nature of the model meant that alternative scenarios - such as changes in police behaviour or resourcing - could be run in order to explore the potential effect of policy interventions.

A second example explored in the ENFOLDing project was maritime piracy. At the time, little was known about spatial patterns of maritime piracy, but the problem was escalating, particularly around 
the Gulf of Aden, and estimates suggested that this problem was costing the global economy around $\$ 7$ billion per year (Ploch, 2010). Our initial research thus aimed at describing patterns using a statistical framework, and in Marchione and Johnson (2013) we demonstrated that incidents of Maritime Piracy were not only spatially clustered, but also clustered in space and time, more than would be expected assuming that the timing and location of events were independent. In Marchione, Johnson and Wilson (2014) we built an agent-based model of maritime piracy, using the Gulf of Aden as a case study. In that paper, we used empirical data on shipping activity to model the flow of potential victims (modelling the activity of different types of vessels from different origins, with different risks associated with each) through the Gulf of Aden, and developed rules - based on the available literature - to guide their behaviour and that of pirate (offenders) and naval vessels (capable guardians). Methods were developed to enable us to calibrate (with one sample of data) and compare the model outcomes to (a different sample of) empirical data. While imperfect, due to missing data on naval strength and operating tactics (which were simply not available), the final model provided a good fit to the empirical data and provided insight into the phenomena and a framework for testing naval strategies. Exemplifying the diversity of approaches applied within the project, Marchione \& Wilson (2016) also developed an alternative approach, using a spatial interaction framework rather than an agent-based formulation. In this case, the quantity modelled represented a notion of "threat"; an example of an abstract flow that was developed further in later work relating to military conflict (Baudains et al, 2016).

\section{Migration}

A different strand of the ENFOLDing project focused on migration flows. While this workstream of the project did not examine the direct implications of migration on security, systems of migration can clearly influence crime and security risk. There is little evidence to suggest that migration leads to crime in neighbourhoods (e.g., Nunziata, 2014), but migrants - particularly those without formal documentation - may themselves be vulnerable, creating opportunities for their victimisation, 
including trafficking, extortion, and exploitation (e.g., Newell et al., 2016). Likewise, as will be discussed later in this paper, outbreaks of unrest, including wars, can lead to the displacement of populations who are forced to flee their homes. Understanding to where they flee and why is important for policy makers.

In the ENFOLDing project, an important question concerned the modelling of interregional migration flows within Europe. While some data exist on such flows, much of it was missing for a number of countries and years of interest. To address this, Dennett and Wilson (2013) developed a multi-level spatial interaction model to estimate the missing flows, using the distance between origins and destinations as the key parameter of the model. While the data necessary to establish the "ground truth" for the entire system was not available, data for some countries (including the UK) were. Comparison of the SI model results with the UK data demonstrated a good overall fit for most countries, with R-squared values of $50 \%$ or more. Further analyses suggested that the exclusion of inner London, which offers (for example) substantial job opportunities, improved the model fit further, indicating the role of omitted variables in migrant decision making. A final point to make here about the model presented in Dennett \& Wilson was that they show how it could be used to produce predictions for the flows from the UK to all other countries - a modelling goal that we sought to address in the work we will discuss next.

As discussed above, three of the current authors were successful in acquiring funding from the US Department of Defense's Minerva Research Initiative to develop, amongst other things, spatial interaction models to help understand refugee flows from conflict zones, focusing on the case of Syria, in particular. These models were informed by and drew inspiration from the ENFOLDing project and hence Alan's contributions to understanding flows and the importance of so doing for policy and research. In what follows we describe this aspect of the work in more detail, presenting some initial findings.

\section{BACKGROUND TO FORCED MIGRATION}


Spatial interaction models of forced migration do not fundamentally differ from equivalent models of other flows, including several of the examples already discussed, and indeed regular migration. They both attempt to mathematically describe and explain flows between sets of origins and destinations, taking into account the stocks and characteristics of both. For a model of the displacement caused by the Syrian Civil War beginning in 2011 (and still ongoing in 2019), these flows will - as with other models of migration - be comprised of people, and in particular the millions of Syrians displaced around the world by the conflict. Where this scenario is distinct from others, though, is in the variables expected to explain the flows, due to the particular (and extraordinary) conditions under which forced migration decisions are taken. Drawing from the wider literature (and the available data), we propose 15 factors which might influence Syrian refugee destination choices, and which we will include in our model.

Firstly, and particularly in the case of forced migration, the decision to flee may be sudden, meaning that those affected may have little opportunity to plan and/or will have limited resources available for their trip. Under such constraints, refugees are likely to prefer, or only be capable of, making shorter trips. Furthermore, because the primary motivation for fleeing in such scenarios is likely to be safety (Moore and Shellman 2007; Missirian and Schlenker 2017), refugees are likely to simply prefer taking refuge in the nearest safe haven, which will often be a bordering country. As well as distance, which is common to all spatial interaction approaches, we therefore include an explicit indicator of contiguity. Further to this point, refugees would also be expected to prefer locations where there are fewer perceived security threats. While such dangers can manifest, and be measured in a variety of ways, some of the most significant issues relevant to the current paper are rates of conflict, terrorism, and crime.

Although forced refugee movements occur under highly constrained circumstances, the literature also emphasizes that it will also generally entail a degree of choice. That is, while refugees may flee to the most easily accessible safe area in the first instance, subsequent journeys to future 
destinations may resemble those observed during other forms of migration, such as those motivated by employment or family reunification (Collyer et al. 2012, Davenport et al. 2003, Zimmermann 2009). As such, factors associated with voluntary migration - such as labour market conditions (including average wages and unemployment rates), the size of an ethnically similar diaspora (or colonial ties), and shared language or cultural similarity - are also relevant to refugee destination choices (e.g., Thieleman 2003, Bocker and Havinga 1997) and as such are modelled in what follows.

We also expect that civil liberties and political rights will play an important role in destination choice (Fitzgerald et al. 2014, Neumeyer 2005, Moore and Shellman 2006). Political rights include, for example, the right to form political parties or groups, have fair elections and party competition. Civil liberties include the right to free speech, freedom of the media and the prevalence of the rule of law. Such liberties and rights are important to refugees who were commonly persecuted in their home countries and wish to avoid a similar fate in their new host countries.

Finally, we expect that the likelihood of being granted refugee status will be an important determinant of destination choice. Many states have publicly declared a willingness to observe international law with respect to non-refoulement of those who seek asylum (Moore and Shellman 2006). As such, refugees may expect not to be turned away immediately when seeking asylum in these countries. However, refugees are likely to seek destinations where there is a higher probability of attaining formal asylum status in the longer-term, which affords them greater rights and a lower risk of deportation.

In what follows, we test the influence of each of these factors (also summarised in Table 1) briefly discussed here have on refugee flows from Syria. In the sections that follow, we describe the methods adopted, including the data analysed and the specific form of the spatial interaction model used, and then present our findings. 


\section{METHODS}

Data

For this analysis of the effects of the Syrian Civil War on migration, data on the flows of official refugees $^{2}$ from Syria are taken for the years 2011 to 2016 from data provided by the United Nations High Commissioner for Refugees [UNHCR]. It is worth noting that these data exclude those who are internally displaced within Syria, of whom there are at least 6 million people. While such displacement is an important component of the forced migration phenomenon, the inconsistency of data between this and the international component - the sources, and methods used, differ ${ }^{3}-$ represents a barrier to their modelling in an integrated way, and so here we consider only the international case. Furthermore, due to limitations in the temporal resolution of the data regarding country characteristics (see below), our models relate to the cumulative total of refugees (20112016) rather than the yearly totals. Lastly, as the data for 2017 and 2018 are either unavailable (the latter) or small counts are anonymised (the former), only data up to 2016 are used.

Refugee flows could be modelled at a range of spatial scales from towns, to countries, to regions. In this paper, we focus on flows at the country level ${ }^{4}$, which is the spatial unit of analysis for which data

${ }^{2}$ In accordance with the UNHCR and the 1951 and 1967 United Nation Conventions and the 1969 Organisation of African Unity Convention, refugees are defined as:

- " "[persons] who, owing to well-founded fear of being persecuted for reasons of race, religion, nationality, membership of a particular social group or political opinion, is outside the country of his nationality and is unable or, owing to such fear, is unwilling to avail himself of the protection of that country, or who, not having a nationality and being outside the country of his former habitual residence as a result of such events is unable or, owing to such fear, is unwilling to return to it"; or

- "[persons] who, owing to external aggression, occupation, foreign domination or events seriously disturbing public order in either part or the whole of his country of origin or nationality, is compelled to leave his place of habitual residence in order to seek refuge in another place outside his country of origin or nationality".

${ }^{3}$ Such differences may substantially affect the estimates of flows and may include the criteria used to classify or define refugees, the sampling method applied, or the data used, the time period over which data are collected, and so on.

${ }^{4}$ We employ the most inclusive definition of 'country', as offered by the International Organization for Standardization's 3166-1 standard, which is an internationally-recognised standard for designating countries. 
are generated by the UNHCR. However, from those countries available, we excluded a number of countries (in addition to Syria) for the following two reasons. First, some of the listed territories had very few (e.g., Antarctica) or no (e.g., Bouvet Island) residents and as such were not commonly referred to as countries, nor expected to be considered as destination choices for those fleeing Syria. Specifically, 63 territories with populations of less than 250,000 (which collectively accounted for only $0.001 \%$ of all Syrian refugees) were removed as possible destination choices from the model. Second, data were unavailable for one or more of the independent variables of interest for 83 countries. Again, these countries accounted for very few Syrian refugees (in this case $0.2 \%$ of the total). After these exclusions, 102 possible destination countries remained, with our dataset containing independent variables for all ${ }^{5}$. Cumulatively, these countries accounted for $84 \%$ of the world's population (excluding Syria). Figure 1 shows the cumulative observed flows for each country included in the analysis.

Table 1 provides summary statistics for each of the variables analysed, a brief description of how they were derived, and their provenance. A number of points are worth noting. First is the fact that distance is measured and tested in two ways. In one version, it is measured using the spatial distance between the capital city of each country and the capital city (Damascus) of Syria. However, to account for the diminishing effects of longer distances, we also include a second version, obtained by taking the natural logarithm of the distance, and we examine the use of both these alternatives. A further point relates to the measurement of crime, which is hampered by the absence of a universal

Unlike many other designations, this scheme identifies countries where there are populations occupying territories. A such, it does not require minimum population thresholds or diplomatic recognition to designate 'country' status.

${ }^{5}$ Analyses were also conducted that included the 62 countries that had up to two missing values. For these countries, the missing data were imputed through estimation, such as taking the mean of the values of that country's nearest neighbours. These analyses revealed the same pattern of results as those reported below and hence are discussed no further. 
definition (legal or otherwise) and its variation both between countries and over time. Moreover, crime 